\title{
Thermodynamic Properties of New Superconductivity Type I Clathrate Compound $\mathrm{Ba}_{8} \mathrm{Si}_{46}$
}

\author{
Wei Zhang*1,2,a , Qingyun Chen ${ }^{1}$, Bin $\mathrm{Li}^{1}$ \\ ${ }^{1}$ Southwest University of science and technology, \\ Mianyang, Sichuan, 610064, China \\ ${ }^{2}$ Laboratory for Shock Wave and Detonation Physics Research, \\ Institute of Fluid Physics, Chinese Academy of Engineering Physics, \\ Mianyang 621900, China \\ azwhys@qq.com
}

\begin{abstract}
The ground state properties and equation of state of the silicon clathrate $\mathrm{Si}_{46}$ intercalated by $\mathrm{Ba}$ atoms are investigated by first-principles calculations based on the plane-wave basis set with the generalized gradient approximation (GGA) for exchange and correlation. Some thermodynamic properties including the heat capacity, the thermal expansion coefficient, Debye temperature and the Grüneisen parameter for $\mathrm{Ba}_{8} \mathrm{Si}_{46}$ are obtained. Moreover, the effects of high pressure on these thermodynamic properties are also investigated which will be very helpful for a synthesis of these clathrates in experiments under high pressure and high temperature condition.
\end{abstract}

Keywords-thermodynamic properties; density functional theory; clathrate compound

\section{INTRODUCTION}

The type-I silicon-clathrates intercalated by $\mathrm{Ba}$ atoms comprised by face-shared polyhedral cages, have the same structure as the methane hydrate $\left(\mathrm{CH}_{4}\right)_{8}\left(\mathrm{H}_{2} \mathrm{O}_{46}[1]\right.$. In the cubic cell of $\mathrm{Ba}_{8} \mathrm{Si}_{46}$, the unique $\mathrm{Si}$ atoms are located at three distinct Wyckoff symmetry sites: Si 6c, Si 16 i and Si $24 \mathrm{k}$. The $\mathrm{Ba}$ atoms are assumed to locate at the centers of two small $\mathrm{Si}_{20}$ cages and six large $\mathrm{Si}_{24}$ cages corresponding to $2 \mathrm{a}$ and $6 \mathrm{~d}$ sites respectively. Such silicon compounds intercalated by $\mathrm{Na}$ atoms were first synthesized by Cros et al. early in 1965 [2,3], and they back to the line of sight recently since the discovery of superconductivity in intercalated clathrate $\mathrm{Ba}_{\mathrm{x}} \mathrm{Na}_{\mathrm{y}} \mathrm{Si}_{46}$ [4]. Soon afterwards, due to their potential applications range from superconductivity[5], large gap semiconductors[6], optoelectronics[7], thermoelectrics[8,9], they become the subject of active experimental and theoretical study. The most known method to synthesize such kind of silicon clathrates is under deep vacuum or at ambient pressure using Zintl compounds as precursors or through different types of chemical reactions.[2,10]. Since the first high-pressure synthesis of type-I $\mathrm{Ba}_{8} \mathrm{Si}_{46}$ clathrate was realized in 2000 by the group of Yamanaka [11] in a multi-anvil press, more and more clathrate compounds were synthesized using high pressure techniques[12,13]. However, as declared in the reference [14], a well-controlled clathrate compounds crystal growth need the knowledge of the $\mathrm{p}-\mathrm{T}$ domains for thermodynamic stability and the possibility for equilibrium crystal growth, which are still wanting. So, in this letter, we employ first principle calculation combine with quasi-harmonic Debye model to investigate the thermodynamic properties of $\mathrm{Ba}_{8} \mathrm{Si}_{46}$ in order to offer some beneficial theoretical reference for experiments.

\section{THEORETICAL METHODS}

In the electronic structure calculations, the ultrasoft pseudopotentials introduced by Vanderbilt [16] have been employed for all the ion-electron interaction, together with the generalized gradient approximation (GGA) [17] for the exchange-correlation function. A plane-wave basis set with energy cut-off $600 \mathrm{eV}$ is applied. Pseudo atomic calculations are performed for $\mathrm{Ba}(5 \mathrm{~s}, 5 \mathrm{p}, 6 \mathrm{~s})$ and $\mathrm{Si}(3 \mathrm{~s}$, $3 p$ ). For the Brillouin-zone sampling, we use the $6 \times 6 \times 6$ Monkhorst-Pack mesh. The self-consistent convergence of the total energy is $10^{-6} \mathrm{eV} /$ atom. All these total energy electronic structure calculations are implemented through Cambridge Serial Total Energy Package (CASTEP) code $[18,19]$.

To investigate the thermodynamic properties of $\mathrm{Ba}_{8} \mathrm{Si}_{46}$, we apply the quasi-harmonic Debye model [20], in which the phononic effect is considered, and the non-equilibrium Gibbs function $G^{*}(V ; P, T)$ takes the form of

$$
G^{*}(V ; P, T)=E(V)+P V+A_{V i b}(\Theta(V) ; T)
$$

where $E(V)$ is the total energy per unit cell, $P V$ corresponds to the constant hydrostatic pressure condition, $\Theta(V)$ is the Debye temperature, and the vibrational contribution $A_{v i b}$ can be written as

$$
A_{V i b}(\Theta ; T)=n k T\left[\frac{9}{8} \frac{\Theta}{T}+3 \ln \left(1-e^{-\Theta / T}\right)-D(\Theta / T)\right]
$$

where the $D(\Theta / T)$ represents the Debye integral, $n$ is the number of atoms per formucla unit. The Debye temperature $\Theta$ expressed by

$\Theta=\frac{\hbar}{\mathrm{K}}\left[6 \pi^{2} V^{1 / 2} n\right]^{1 / 3} f(\delta) \sqrt{\frac{B_{S}}{M}}$ 
where $M$ is the molecular mass per formula unit, $B_{\mathrm{S}}$ is the adiabatic bulk modulus, $\sigma$ is the Poisson ratio and $f$ $(\sigma)$ is given by

$$
f(\sigma)=\left\{3\left[2\left(\frac{2}{3} \frac{1+\sigma}{1-2 \sigma}\right)^{\frac{3}{2}}+\left(\frac{1}{3} \frac{1+\sigma}{1-\sigma}\right)^{\frac{3}{2}}\right]^{-1}\right\}^{\frac{1}{3}}
$$

By solving the following equation with respect to $V$

$$
\left(\frac{\partial G^{*}(V ; P, T)}{\partial V}\right)_{P, T}=0
$$

one could obtain the thermal expansion coefficient $\alpha$ as follows

$$
\alpha=\gamma C_{V} /\left(B_{T} V\right)
$$

where the isothermal bulk modulus $B_{\mathrm{T}}$, the heat capacity $C_{\mathrm{V}}$ and the Grüneisen parameter $\gamma$ are expressed as

$$
\begin{aligned}
& B_{T}(P, T)=V\left[\frac{\partial^{2} G^{*}(V ; P, T)}{\partial^{2} V^{2}}\right]_{P, T} \\
& C_{V}=3 n k\left[4 D(\Theta / T)-\frac{3 \Theta / T}{e^{\Theta / T}-1}\right] \\
& \gamma=\frac{d \ln \Theta(V)}{d \ln V}
\end{aligned}
$$

\section{RESULTS AND DISCUSSION}

For clathrate compounds $\mathrm{Ba}_{8} \mathrm{Si}_{46}$, a series of lattice constants are set to obtain the total energy $E$ and the corresponding primitive cell volume $V$ through GGA schemes, and then the obtained $E-V$ data are fitted to the Birch-Murnaghan equation of state (EOS) [21]. From the EOS we find that when the pressure applied to $30 \mathrm{GPa}$, the volume of clathrate compound $\mathrm{Ba}_{8} \mathrm{Si}_{46}$ just reduced about $20 \%$ which show this compound is not easy to compressed. The obtained equilibrium lattice constant $a$ equals to $10.375 \AA$, zero-pressure bulk modulus $B_{0}$ and its pressure derivative $B_{0}{ }^{\prime}$ are $75.5 \mathrm{GPa}$ and 5.2 respectively.

We plot the dependence of both heat capacity at constant volume $C_{V}$ and heat capacity at constant pressure $C_{p}$ on temperature from 0 to $700 \mathrm{~K}$ in Figure 2. The calculated heat capacity $C_{p}$ and $C_{V}$ at ambient condition are 1317.9 $\mathrm{J} \mathrm{Mol}^{-1} \mathrm{~K}^{-1}$ and $1255.6 \mathrm{~J} \mathrm{Mol}^{-1} \mathrm{~K}^{-1}$ respectively. The experimental heat capacity is that at constant pressure, i.e., $\mathrm{C}_{p}$, but the more meaningful quantity to discuss lattice dynamics is $C_{V}$. For a cubic solid, the relationship between them can be expressed by $C_{\mathrm{p}}-C_{\mathrm{v}}=K T V\left(\alpha_{\mathrm{V}}\right)^{2}$. The difference between $C_{\mathrm{p}}$ and $C_{\mathrm{V}}$ is about $4.9 \%$ at room temperature, when the temperature decrease to about 100 $\mathrm{K}$, the difference between then can hardly be found. Moreover, it can be found that when the temperature is below $300 \mathrm{~K}$, the heat capacity $C_{V}$ is strongly dependent on temperature, which is due to the anharmonic approximations. However, under higher temperature, the anharmonic effect on $C_{V}$ is suppressed, and the $C_{V}$ is close to a constant at high temperature.

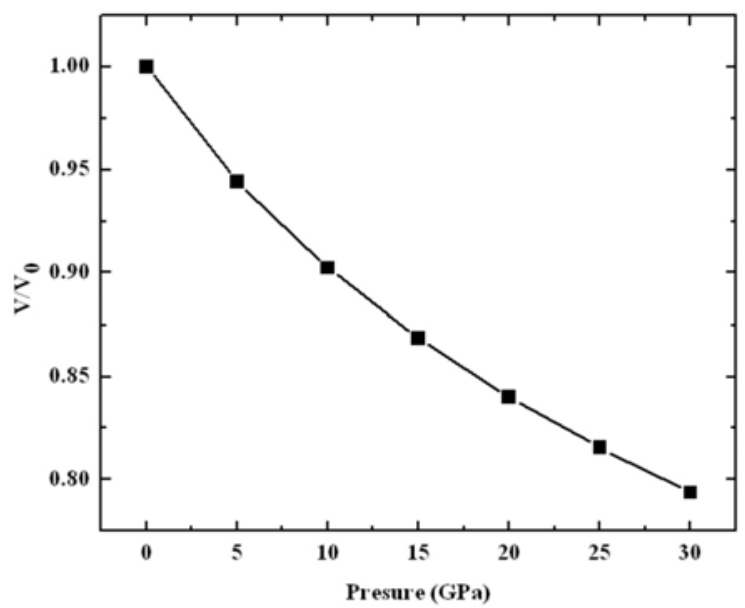

Figure 1. Calculated equation of state of $\mathrm{Ba}_{8} \mathrm{Si}_{46}$

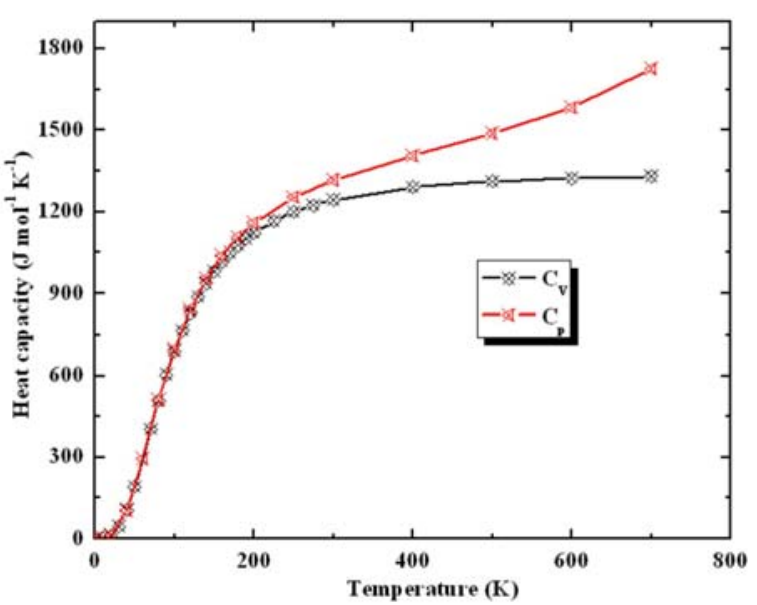

Figure 2. Dependences of specific heat for $\mathrm{Ba}_{8} \mathrm{Si}_{46}$ on temperature

In Figure 3, The temperature dependences of thermal expansion coefficient $\alpha_{v}$ of $\mathrm{Ba}_{8} \mathrm{Si}_{46}$ under different pressures are illustrated. It can be found that the thermal expansion coefficient $\alpha_{v}$ increases with $T^{3}$ at low temperatures and gradually approaches a very slow increase at high temperature. At low temperatures, the effects of pressure on the thermal expansion coefficient $\alpha_{v}$ are very small. The pressure can suppress such thermal expansion obviously. The variation of Grüneisen parameter $\gamma$ with pressure and temperature are displayed in Figure.4, from which it can be found that the Grüneisen parameter $\gamma$ increases as the temperature increases, however, as the pressure enhanced, the effect of temperature becomes more and more infirm because at high pressure, the increasing trend becomes rather gentler. 


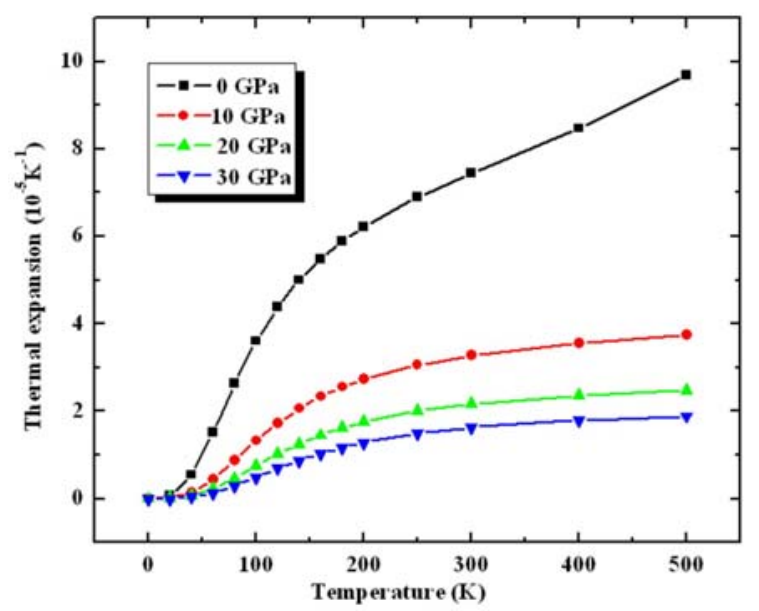

Figure 3. Temperature dependences of thermal expansion coefficient for $\mathrm{Ba}_{8} \mathrm{Si}_{46}$ under different pressures

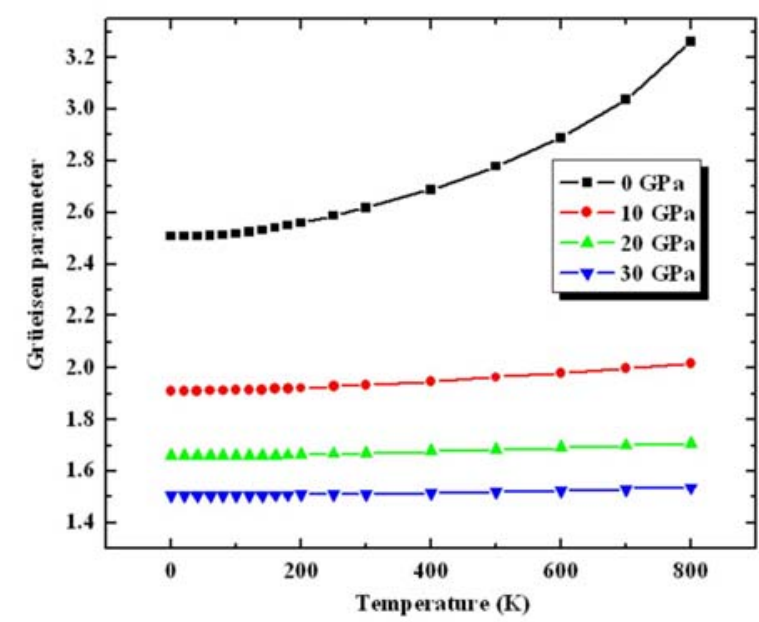

Figure 4. Temperature dependences of the Grüneisen parameter for $\mathrm{Ba}_{8} \mathrm{Si}_{46}$ under different pressures

The dependence of Debye temperature on temperature under different pressures are shown in Figure 5. The value of Debye temperature can represents inter-atomic binding forces thus it is related to hardness of material, in general, a higher Debye temperature indicates a higher hardness[22]. The dependence of Debye temperature on temperature at zero pressure shows a soften of the $\mathrm{Ba}_{8} \mathrm{Si}_{46}$ crystal and the pressure can suppress such effect. At room temperature, the calculated Debye temperature $\Theta_{\mathrm{D}}$ is $395 \mathrm{~K}$. From Figure 5, it can be found that the pressure has a more distinct effect on $\Theta_{\mathrm{D}}$ than temperature. When the pressure applied to $30 \mathrm{GPa}$, although the temperature enhanced, the Debye temperature is almost a constant.

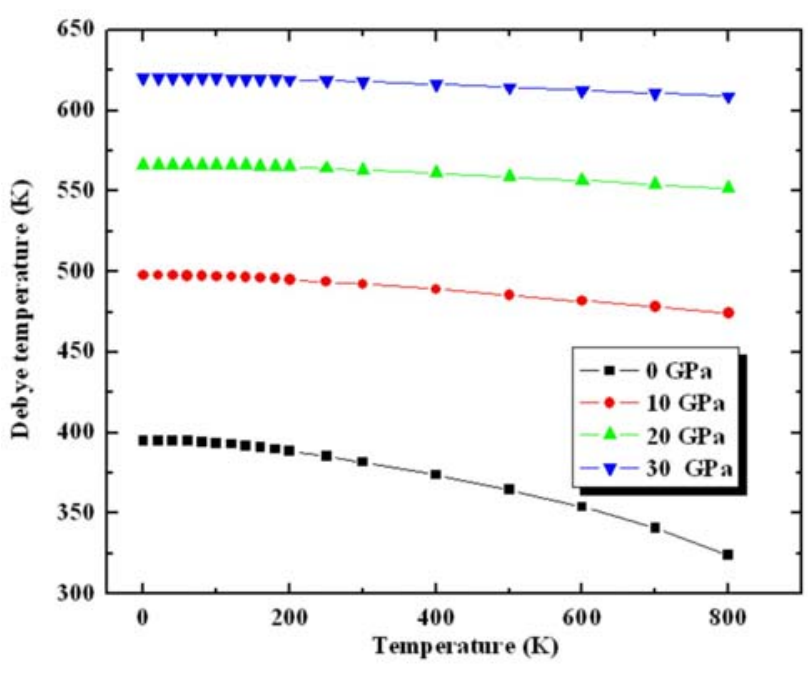

Figure 5. The dependence of Debye temperature on temperature at 0,10 , 20 and $30 \mathrm{GPa}$

\section{SUMMARY}

In summary, the thermodynamics properties of silicon clathrate intercalated by $\mathrm{Ba}$ atoms are obtained by first-principles calculations combine with quasi-harmonic Debye model. The EOS, heat capacity, thermal expansion coefficient and Debye temperature are calculated. The dependence of them pressure on are also discussed, it is found that the effects of pressure on thermal expansion of $\mathrm{Ba}_{8} \mathrm{Si}_{46}$ are very small at low temperatures, but as the temperature increases the effects become more prominent. As for the variation of Grüneisen parameter, pressure plays a dominant role.

\section{ACKNOWLEDGMENT}

This work was supported by the National Natural Science Foundation of China under Grant No.11347134 and 11304254, and by the Doctor Foundation of Southwest University of Science and Technology under Grant No. $13 \mathrm{zx} 7125$.

\section{CORRESPONDING AUTHOR}

The corresponding author of this paper is Zhang Wei, whose email is zwphys@qq.com, mobile phone number is +8618780573558 .

\section{REFERENCES}

[1] E. D. Sloan: Clathrate Hydrates of Natural Gases (Marcel Dekker, New York, 1998).

[2] C. Cros, M. Pouchard and P.Hagenmuller, C.R.Acad.Sci., 260, 4764 (1965).

[3] J.S.Kasper,P.HagenmullerandM.Pouchard, Science ,150 1713(1965).

[4] H.Kawaji,H.Horie,S.Yamanaka,and M.Ishikawa, Phys.Rev.Lett., 74,1427 (1995).

[5] HerrmannRFW, TanigakiK, Kuroshima $\mathrm{S}$ and Suematsu H, Chem.Phys.Lett. ,283, 29 (1998). 
[6] G. S. Nolas ,J. L. Cohn , G. A. Slack and S. B. Schujman , Appl.Phys.Lett. ,73 178 (1998).

[7] M.Menon, E. Richter and K. Subbaswamy, Phys.Rev. B, 5612290 (1997).

[8] G. A. Slack, Mater.Res.Soc.Symp.Proc., 478, 47 (1997).

[9] J. Martin ,H.Wang and G. S. Nolas , Appl.Phys.Lett., 92, 222110 (2008).

[10] M. Beekman, ; G. S. Nolas, J. Mater. Chem., 18, 842 (2008),

[11] S.Yamanaka, E. Enishi, H. Fukuoka et al., Inorg. Chem., 39, 56 (2000).

[12] P. Toulemonde, Ch. Adessi, X. Blase et al., Phys. Rev. B, 71094504 (2005).

[13] R. Viennois, P. Toulemonde, C. Paulsen et al., J. Phys.: Condens. Matter ,17, L311 (2005)
[14] O. O. Kurakevych, T. A. Strobel, D. Y. Kim, T. Muramatsu and V. V. Struzhkin, Cryst. Growth Des., 13, 303 (2013).

[15] S. Stefanoski, M. C. Blosser, and G. S. Nolas, Cryst. Growth Des., 13, 195, (2013).

[16] Vanderbilt D. Phys. Rev. B, 41, 7892 (1990).

[17] J. P. Perdew , K.Burke, and M. Ernzerhof , Phys. Rev. Lett., 77, 3865 (1996).

[18] M. Parrinello and A. Rahman, Phys. Rev. Lett., 45, 1196 (1980).

[19] M.Parrinello , A.Rahman, J. Appl. Phys., 52, 7182 (1981).

[20] Blanco M. A., Francisco E., and Luana V., Comput. Phys. Commun., 158, 57 (2004)

[21] F. Birch, Phys. Rev. ,71, 809 (1947).

[22] P. Ravindran, L. Fast, P.A. Korzhavyl, B. Johansson, J. Wills, O. Eriksson, J. Appl. Phys. 84, 8 (1998). 